\title{
Acknowledgements to the Referees
}

\section{Ophthalmic Research}

\author{
Muntasir Abdulaziz \\ Luis Abegão Pinto \\ Nadiah Abu \\ Faisal Ahmed \\ Csilla Ajtony \\ Nicolas Alejandre \\ Jorge Alio \\ Khaldoon al Nosairy \\ David Alonso-Caneiro \\ Abdullah Alqahtani \\ Albert J. Augustin \\ Célia A. Aveleira \\ Konstantinos Balaskas \\ Piero Barboni \\ Andreas Bringmann \\ Monika Buraczynska \\ Elisa Campos \\ Tommy Chan \\ Irini Chatziralli \\ Itay Chowers \\ Colin Clement \\ Laura Crawley \\ Fernando Cruz-Gonzalez \\ Lajos Csincsik \\ Adrienne Csutak \\ Christine A. Curcio \\ Benjamin Davis \\ Marci DeRamus \\ Fabiana D'Esposito \\ Susov Dhakal \\ Ali Dirani \\ Nobuyuki Ebihara \\ Amal M. Elbendary \\ Michel Farah \\ Rosa Fernandes \\ Antonio Ferreras \\ João Figueira \\ Francisco Figueiredo \\ Kenneth Fong \\ Alex Fonollosa \\ Cláudio Franco \\ Samantha Fraser-Bell \\ Andreas Frings \\ Lars Fritsche \\ Ahmed Galal \\ Rajendra K. Gangalum
}

Clare Gilbert

Stewart Gillan

Henrique Girão

David Goldblum

Gnanaguru Gopalan

Carolina P.B. Gracitelli

John Grigg

Christian Grimm

Yan Guex-Crosier

Ratna Gupta

Na'ama Hammel

Hideaki Hara

Michael Hauser

Tjebo Heeren

Nino Hirnschall

Rumana Hussain

Pierluigi Iacono

Maiko Inoue

Ugo Introini

Takeshi Iwase

Vesna Jaksic

Georgia Kaidonis

Evgenia Kanonidou

Min Kim

Tolga Kocaturk

Christos Kroupis

Hee Jung Kwon

Antoine Labbe

Timothy Y. Lai

Liang Liu

Francesco Loperfido

Kristian Lundberg

Lyubomyr Lytvynchuk

Shanu Markand

Gottfried Martin

Tammy Martin

Antonio Mateo

Milka Mavija

Manuel Mayr

Carla Medeiros

Marco D. Medeiros

Frances Meier-Gibbons

Jesus Merayo-Lloves

Zofia Michalewska

Kensaku Miyake

Alyson Muldrew
Ana Maria Muñoz

Joaquim Murta

Eduardo Normando

Sina Noshad

Katarzyna Nowomiejska

Colm O'Brien

Jaeryung $\mathrm{Oh}$

Neville Osborne

Leonidas Papazisis

Dong Ho Park

Marta Pazos

Eli Peli

Serge A. Picaud

Tiago Prata

Heiko Pult

Thomas Ritter

Andreia Rosa

Sylvain Roy

Ramon Ruiz Mesa

Maite Sainz de la Maza

Ferenc Sallo

Juliana Sallum

Ana Raquel Santiago

Mariacristina Savastano

Michal Schaap Fogler

Eamon Sharkawi

Harmanjit Singh

Mário Soares

Werner Spileers

Andreas Stahl

Knut Stieger

Brijesh Takkar

Alicia Valverde

Pradeep Venkatesh

Daniel Vila Solà

Simon von Spreckelsen

Stela Vujosevic

Cathy Williams

Yasuo Yanagi

T. Yasukawa

Munemitsu Yoshikawa

Alon Zahavi

Michael Zeschnigk 